In some circles the idea still persists that in cleaning a picture there is a positive virtue in avoiding the complete removal of the old varnish, this being claimed as a mild form of treatment. It is a specious argument, and the practice is only in favour in commercial ateliers where saving in cost is a factor or where there is lack of confidence or ability on the part of the restorer. It is to be condemned for the following reasons. First, because the use of feeble solvents necessitates using friction, and the rubbing of the surface of the painting tends to remove ridges of paint in the brush-work or crackle system. The hollows of the impasto and the cracquelure tend, inevitably, to become filled with a brown residue consisting of the least soluble constituents of the old varnish. There is this, also, to be said, that a picture so treated will speedily darken to the point of requiring re-cleaning, and the net result is that the incidence of cleaning is increased. Residues of the old varnish may well promote crystallization of the new. The practice of treating pictures along these lines cannot be too strongly condemned. One admits, however, with reluctance, that circumstances may occasionally require a compromise to be made. When this is so it can only be in favour of dark passages of colour : from light pigments and bright colour, all of the old varnish must be completely removed, and the degree of success of the operation can be followed in the laboratory by examining the painting under screened ultra-violet illumination, when the old resin residues are seen to fluoresce unmistakably.

\section{Atmosphere and Stability}

While pictures are re-conditioned by cleaning and restoration, their preservation is entirely dependent on the conditions of humidity and temperature in the picture gallery.

When humidity and temperature are uncontrolled, the day and night changes occasion rhythmic movements in the panels and canvases. These may be slight; but in the aggregate they have a deleterious effect on the stability of the paint layer. A consequence is the appearance of blisters in the paint, caused usually by cleavage between the ground and the support, and in the course of a year many are discovered and 'laid' by competent craftsmen. If undiscovered, the next stage is fracture and exfoliation of the paint, necessitating more serious treatment. Extreme atmospheric conditions or changes of condition are liable to cause catastrophe, such as disruption of the constituent planks of Holbein's "Ambassadors" panel (February 1895), or the splitting of Rubens" great "Château de Ste日n" (January 29, 1947).

It took experience of the war-time repository to bring out the fact that under controlled conditions of humidity and temperature ('conditioned air') no blisters form ; there was practically no maintenance work of this kind required at Manod Quarry ${ }^{2}$ in the course of years. The moral is obvious and of the greatest significance to the preservation of paintings : conditioned air must be accepted as essential for picture galleries. In the national collection, which is so rich in large Italian panels, a grave responsibility rests upon all who realize what is at stake. This is understood and accepted by the authorities, who, with the help of the Ministry of Works, have improvised a 'hospital' behind the scenes, and here by the aid of primitive controls the relative humidity can at least be held in check. By this means a floating population averaging some thirty 'ailing' pictures is saved, temporarily, from exposure to the more extreme climatic conditions of the public galleries.

It is with relief that we learn that a scheme for installing conditioned air in the National Gallery is in being. The apparatus exists already on the site. Plans have been approved for the first experimental section, and we can look forward to the coming of an era-let us hope it may be soon-when the building will be worthy of its great collection of pictures, cleaned by the enlightened methods of to-day, so that all in their generation may enjoy the work of the old masters as they themselves would have wished.

1 "La Conservation des Peintures" (L'Institut International de Co-

Rawlins, F. I. G., Nature, 151, 123 (1943).

\section{HYPOTHETICAL ALTERNATIVE ENERGY SOURCES FOR THE 'SECOND MESON' EVENTS}

\author{
By Dr. F. C. FRANK, O.B.E.
}

H. H. Wills Physical Laboratory, University of Bristol

T the paper by Lattes, Occhialini and Powell*, it 1 is shown that cosmic ray mesons coming to the end of their track in a photographic emulsion rather frequently give rise to a secondary meson of kinetic energy about $4 \mathrm{MeV}$., and no other visible particle. This observation appears to demand for its interpretation the existence of two sorts of meson, the source of the observed kinetic energy of the secondary residing in the mass difference between the two. In view of the importance of this conclusion, we have tried to consider all reasonably imaginable processes to explain the observations without introducing a new elementary particle; processes, that is, in which the energy is derived from the material of the photographic emulsion instead of from the meson. Reasons are given below for the confident rejection of all such processes.

It is concluded that there is no energy to be gained, from elements present in substantial quantity in the photographic emulsion, from the following processes :

(a) Induced $\beta$ - or $K$-capture processes : on grounds of known mass defects for the lighter nuclei, and systematic principles for the heavier nuclei present. There should be energy (though not enough to explain the observations) to gain from such processes from $\mathrm{K}^{40}, \mathrm{Rb}^{87}, \mathrm{Lu}^{178}, \mathrm{Os}^{187}$ and from one each of the pairs $\mathrm{In}^{113}, \mathrm{Cd}^{113}, \mathrm{Sn}^{115}, \mathrm{In}^{115}, \mathrm{Te}^{123}, \mathrm{Sb}^{123}$; none of these is present in substantial quantity. It is improbable that any natural nucleus possesses a more stable adjacent isobar not discovered in Nature.

(b) Induced emission of single nucleons: on grounds of known mass defect for the lighter nuclei and systematic principles for heavier ones; such processes, from naturally existing nuclei, always absorb energy.

(c) Induced $\alpha$-emission : excluded for the lighter nuclei by known mass-defects. Not excluded for the heavier nuclei, since mass defects are not known with sufficient accuracy, and by the Geiger-Nuttall rela-

See Nature, 160, 453 and 486 (1947). 
tion the life-times of low-energy $\alpha$-emitters are too great for detection. However, with no heavier nuclei than silver present, energies greater than about $2 \mathrm{MeV}$. are excluded by this consideration, because they would lead to spontaneous decay at a detectable rate. Moreover, the Coulomb repulsion from a medium-heavy nucleus would assuredly give the $\alpha$-particle sufficient energy to make a visible track.

(d) Induced fission : once again Coulomb repulsion would assuredly produce a visible track from the fission fragments, and it is inherently improbable that a meson could activate the process, say in silver, the only element which comes in question from the point of view of availability of energy.

(e) Processes with change of charge by 2, for example, of the type

$$
A t_{Z}^{A}+Y^{-} \rightarrow A t_{Z-2}^{A}+Y^{+},
$$

where $Y^{-}$and $Y^{+}$denote negative and positive mesons. It is to be expected that this process, if it can occur, would yield energy from about half the nuclei which possess stable isobars of charge diminished by 2. In the case of $\mathrm{Zn}^{64}$ going to $\mathrm{Ni}^{84}$, measured mass defects indicate an energy yield of $9 \cdot 8 \pm 3 \mathrm{MeV}$. The conditions for this process are not satisfied by any nucleus present in substantial quantity in the photographic emulsion, namely, by isotopes of hydrogen, boron, carbon, nitrogen, oxygen, silver, bromine, iodine and sulphur; of these only $S_{18}^{38}$, the rarest $(0.016$ per cent) of the isotopes of sulphur, which is itself present only in traces, has a known stable isobar $A_{18}^{38}$. That is in the wrong direction, requiring the improbable converse process starting with a positive meson.

$(f)$ Induced decay of naturally persisting nuclear isomers : this would imply the existence of isomers with life-times about $10^{12}$ times as great, and stored energies about ten times as great, as any yet knowna very improbable combination.

This seems to exhaust the possibilities among processes of nuclear degradation. On the other hand, it needs more consideration to exclude the possibility of processes of nuclear build-up, in which a proton is added to some other nucleus: such processes are almost all exothermic. The possibility of such processes arises from the special properties of the combination of proton and negative meson, which may be called a mesonic hydrogen atom, or an excited neutron, at choice.

A meson stopping in the emulsion loses kinetic energy from, say, $100,000 \mathrm{eV}$. down to $2,000 \mathrm{eV}$. in a fraction of a micron. This may occur either in silver bromide, or in gelatine, with comparable probability. In the latter case it is very likely to be in the neighbourhood of a proton, of which there are about twice as many as all other nuclei together in the gelatine. It should then, at first, enter a hydrogen-like orbit about the proton. The resulting compact neutral atom (radius, in the 'Coulombic ground-state', about $1 / 200$ of the Bohr radius, thus $2 \cdot 6 \times 10^{-11} \mathrm{~cm}$., and binding energy about 200 times that of a hydrogen atom, thus $2,700 \mathrm{eV}$.) should appear, from distances exceeding $10^{-10} \mathrm{~cm}$., like a slow neutron. It should be able to pass freely through the electronic clouds of other atoms, and approach close to nuclei, without Coulomb repulsion. Indeed, the polarization of the mesonic atom in the field of a second nucleus should lead to an initial attraction.

The most probable next step would appear to be the capture of the meson by the nucleus of higher charge, and repulsion of the proton. There should, however, be a non-vanishing probability that the proton is captured, and the meson expelled. In one case at least, namely, that in which the second nucleus is a deuteron, the probability of the alternative reaction should be high. For by analogy with the hydrogen molecule-ion, $\mathrm{H}_{2}{ }^{-}$, the combination of deuteron, proton and meson should be stable in respect of Coulombic interactions with an internuclear separation of $10^{-8} / 200=5 \times 10^{-11} \mathrm{~cm}$. At this distance, with a very low Gamow barrier, the two nuclei should very readily unite, the meson acquiring kinetic energy by what may be described as internal conversion'. Thus, formally, we should have the reaction :

$$
\begin{aligned}
& \mathrm{H}_{1}^{1}\left(e^{-}\right)+Y^{-} \rightarrow \mathrm{H}_{1}^{1}\left(Y^{-}\right)+e^{-}+2,700 \mathrm{\theta V} . \\
& \mathrm{H}_{1}^{2}\left(Y^{-}\right)+D_{1}^{2} \rightarrow \mathrm{H}_{1}^{1} D_{1}^{2}\left(Y^{-}\right)+500 \mathrm{eV} \text {. } \\
& \mathrm{H}_{1}^{1} D_{1}^{2}\left(Y^{-}\right) \quad \rightarrow \mathrm{He}_{2}^{3}+Y^{-}+5.46 \mathrm{MeV} \text {. }
\end{aligned}
$$

It is highly improbable that the small amount of deuterium present in the normal emulsion could account for the observed phenomena. The corresponding process with a second proton (forming a deuteron, since $\mathrm{He}^{2}$ is not stable) requires the emission of a positron and neutrino which, between them, would take most of the $1.43 \mathrm{MeV}$. available, which is in any case insufficient. With heavier nuclei the details of interaction are more complicated, since, among other considerations, there will only be binding in the molecule-ion while it is in an excited state.

The mesonic hydrogen atom will be attracted to a second nucleus by virtue of polarization. Some energy can be dissipated to form the molecular combination, by Auger effect, which will cease to operate when the nuclei and meson are well within the $K$-shell of electrons. The combination dissociates when the meson falls by a radiative transition into a level in which it is concentrated around the nucleus of higher charge. The time taken for this radiation should be of the order $\left(h c / e^{2}\right)(\lambda / r)^{2}(1 / \omega)$, which is about $10^{8} Z^{-2}$ periods of the emitted radiation (as in hydrogen-like spectra) whether we are dealing with mesons or electrons. The characteristic frequency should be 200 times that of the corresponding electronic spectrum, that is, say, $10^{18} Z^{2}$, and the frequency of molecular vibration $(200)^{3 / 2}$ times that of electronically bound hydrogen compounds, that is, about $3 \times 10^{17}$. Hence, we estimate the life-time of the bound state as about $10^{-10} Z^{-4}$ sec., or about $3 \times 10^{7} Z^{-4}$ periods of oscillation of the mesonic molecule-ion.

The Gamow barrier to be penetrated by the proton extends from the internuclear separation in the excited molecule-ion, $r_{1}$, to the distance at which the nuclei make contact, $r_{0}$. The meson still provides some screening within this distance, and we take the potential energy to be $E=(Z-1) e^{2} / r$. We estimate $r_{1}$ as that distance at which the polarizing action of the second nucleus, on the mesonic hydrogen, regarded as linear with field strength, produces an equivalent displacement of the meson equal to its Bohr radius, $a_{Y}=2.6 \times 10^{-11} \mathrm{~cm}$. Thus, $r_{1}=$ $3 a_{Y} \sqrt{Z / 2}=5.5 \times 10^{-11} Z^{1 / 2} \mathrm{~cm} .: r_{0}$ we take as $1.45 \times 10^{-13}\left(A^{1 / 3}+1\right) \mathrm{cm}$., where $A$ is the mass number of the second nucleus. Then, if we write $\lambda^{*}=h / \sqrt{2 M\left(E-E_{0}\right)}$ (where $M$ is the mass of the proton) and neglect the initial kinetic energy $E_{0}$, the transparency of the barrier to a proton wave should be of the order 


$$
\begin{gathered}
\exp \left\{-4 \pi \int_{r_{\theta}}^{r_{1}}\left(1 / \lambda^{*}\right) d r\right\} \\
=\exp \left\{\frac{-8 \pi e \sqrt{2 M(Z-1)}}{h}\left(r_{1}^{1 / 2}-r_{0}{ }^{1 / 2}\right)\right\} \\
=\exp \left\{-3 \cdot 33 \sqrt{Z-1}\left(7 \cdot 4 Z^{1 / 4}-0 \cdot 4 \sqrt{A^{1 / 3}+1}\right)\right\},
\end{gathered}
$$

which is about $10^{-27}$ for $B_{5}^{11}$. This, subject to considerable error, for example, from the uncertainty of $r_{1}$, expresses the probability that the proton will penetrate the barrier in one attempt. Multiplying by the number of oscillations before dissociation, we find the probability of penetration before dissociation to be $10^{-21}$ in this case. This result may well be in error by several powers of 10 , but it appears that if the model is at all relevant, the chance of penetration is insignificant for all nuclei beyond the devteron. The same calculation for the latter, neglecting the inner screening and so writing 1 in place of $\sqrt{Z-1}$, gives a penetration probability of $10^{-9}$ per vibration, so that penetration may occur in about $10^{-8} \mathrm{sec}$.

Specific forces of the meson have been disregarded. There is an obvious chance that it will suffer nuclear capture or destruction before the two nuclei can interact, the probability of which is reduced by the fact that the meson is in excited states throughout the period significant for this process. If, on the other hand, the mesonic forces lead to some closer union of proton and meson than we have considered (differing from a neutron), it might well react with any nucleus.

The energy available from proton adhesion is small $(1.96$ and $0.51 \mathrm{MeV}$. respectively) for the 'saturated' nuclei $\mathrm{C}_{\theta}^{12}$ and $\mathrm{O}_{8}^{18}$. For nearly all other nuclei it is $5 \mathrm{MeV}$. or more. The next most common nucleus in the gelatine is $\mathrm{N}_{7}^{14}$, yielding $7.3 \mathrm{MeV}$., which would account for the observations if the product, $\mathrm{O}_{8}^{15}$, was formed with an excitation of about $3.5 \mathrm{MeV}$. Boron was also present in these emulsions, and could yield more than enough energy by proton adhesion, but in this case the most likely reactions liberate $\alpha$-particles.

This process can in any case be rejected statistically For a total of 380 mesons observed to stop in the emulsion, four secondary mesons have been observed, with at least $500 \mu$ of secondary track in the $50-\mu$ thickness of the emulsion, and mutually consistent in energy yield. Geometrical probability indicates that about thirty times as many events should have occurred, in which the secondary meson passed out of the emulsion earlier : it could then either go undetected or be indistinguishable from a proton. On the other hand, one should not fail to detect a projected length of $50 \mu$ of the end of a meson track. Hence the four observed secondary mesons are representative of about $120 \pm 60$ (the limits expressing probable error) whereas the 380 mesons observed to stop should represent not more than about 900 actually stopping. (For randomly directed straight tracks ending at random depths in an infinite layer of thickness $H$, the statistical proportion of all tracks which ond in the layer which have horizontally projected ranges within the layer exceeding $R$ is $\left(\sqrt{R^{2} / H^{2}+1}-R / H\right)$. For $R / H=1$, this is 0.414 . For $R / H>1$, it is well approximated by $H / 2 R$, but for $R / H \gg 1$, track curvature will reduce the proportion below this estimate.)

Thus it appears probable that. the production of a $4 \mathrm{MeV}$. secondary meson occurs for $13 \pm 7$ per cent of the mesons stopping in the emulsion. Presumably only about half the mesons are negative, and only about half of these can form mesonic hydrogen atoms (since $\mathrm{H}$ comprises 40 atomic per cent of the whole emulsion): if we suppose the interaction with a second proton is weak, so that the mesonic hydrogen is most likely, and about equally likely, to be attracted to carbon, nitrogen or oxygen ( 1 in 8 of which nuclei, in the gelatine, is $\mathrm{N}^{14}$ ), we have an expectation of about 3 per cent if every encounter of a mesonic hydrogen atom with an $\mathrm{N}^{14}$ nucleus leads to capture of the proton, and on every occasion there is 'internal conversion' of the liberated energy, producing a secondary meson of $4 \mathrm{MeV}$. Unconsidered factors are mostly unfavourable, so that even on the small number of observations it is statistically improbable that they represent this process.

A further consideration is that the simple theory leads us to expect a larger number of easily observable $2 \mathrm{MeV}$. - and possibly of $\frac{1}{2} \mathrm{MeV}$.--secondary mesons deriving their energy from proton adhesion to $\mathrm{C}_{6}^{12}$ and $\mathrm{O}_{8}^{18}$ : though it is a simple and plausible addition to the theory to suppose that these reactions are relatively 'forbidden'.

It should be added that such processes may be of importance in other circumstances ; if it is correct to suppose that mesons can survive for, say, $10^{-8} \mathrm{sec}$. in hydrogen-like orbits about protons, then there is a finite chance that a meson can induce a nuclear build-up reaction, causing the attachment of a proton to a deuteron. However, with two or more cosmic ray mesons in the field, the whole of our observational. knowledge about mesons requires re-examination.

I am indebted for a number of discussions to Dr. H. Fröhlich and Messrs. Lattes, Occhialini and Powell.

Note added in proof. Later observations enable the 'corrected observed' proportion of mesons producing secondaries to be refined from $13 \pm 7$ per cent to $12 \cdot 8 \pm 2 \cdot 5$ per cent $(117 \pm 20$ in $917 \pm 70)$, thus increasing the confidence with which the hypothetical alternative process can be rejected.

\section{OBITUARIES}

\section{Prof. T. David Jones, C.B.E.}

IT is with much regret that we record the tragic death of Prof. Thomas David Jones in a motor accident which occurred outside Amesbury on August 30.

T. David Jones was born of a mining family at Aberyskir, Breconshire, in 1900. He was educated at the Technical College, Swansea, and the University College, Cardiff. After the First World War, during which he served with the 17th Lancers, he resumed his studies at the University of Birmingham. He graduated, and passed the Colliery Managers' Exam. ination in 1922; obtained his M.Sc. in 1923 and the Ph.D. a year later. He was then appointed to the mining research staff directed by the late Prof. J. S. Haldane. In this capacity, he carried out extensive investigations into mining conditions, spontaneous combustion, geothermic gradients and mine lighting.

In 1936, Prof. Jones was appointed to the chair of mining at the University College, Cardiff, and in 1939 he became director of research to the Monmouthshire and South Wales Coal Owners' Association, in which position he was responsible for intensive investigations carried out on the suppression of dust 